\title{
Phase 1/2 Dose-Escalation Study of a GM-CSF- Secreting, Allogeneic, Cellular Immunotherapy for Metastatic Hormone-Refractory Prostate Cancer
}

\author{
Celestia S. Higano, мо ${ }^{1}$ \\ John M. Corman, $\mathrm{MD}^{2}$ \\ David C. Smith, MD ${ }^{3}$ \\ Arthur S. Centeno, $\mathrm{MD}^{4}$ \\ Christopher P. Steidle, MD ${ }^{5}$ \\ Marc Gittleman, MD ${ }^{6}$ \\ Jonathan W. Simons, $\mathrm{MD}^{7}$ \\ Natalie Sacks, ${ }^{8}{ }^{8}$ \\ Junko Aimi, PhD ${ }^{8}$ \\ Eric J. Small, ${ }^{9}{ }^{9}$ \\ ${ }^{1}$ Departments of Oncology and Urology, University \\ of Washington, Seattle, Seattle, Washington. \\ ${ }^{2}$ Floyd and Delores Jones Cancer Center, Virginia \\ Mason Medical Center, Seattle, Washington. \\ ${ }^{3}$ Departments of Internal Medicine and Urology, \\ University of Michigan Medical Center, Ann Arbor, \\ Michigan. \\ ${ }^{4}$ Urology San Antonio, San Antonio, Texas. \\ ${ }^{5}$ Northeast Indiana Research, Fort Wayne, Indiana. \\ ${ }^{6}$ South Florida Medical Research, Aventura, Florida. \\ ${ }^{7}$ Winship Cancer Institute, Emory University School \\ of Medicine, Atlanta, Georgia. \\ ${ }^{8}$ Department of Clinical Research, Cell Genesys, Inc, \\ South San Francisco, California. \\ ${ }^{9}$ Comprehensive Cancer Center, University of Cali- \\ fornia, San Francisco, San Francisco, California.
}

Research Support was provided by Cell Genesys, South San Francisco, California, to each investigator (C.S.H., J.M.C., D.C.S., A.S.C., C.P.S., M.G., J.W.S., E.J.S.).

N. Sacks and J. Aimi are employees of Cell Genesys.

J.W. Simons is a coinventor of 2 issued US patents (US Pat. Nos. 7,226,606 and 7,217,421), has pending applications that are exclusively licensed to and co-owned by Cell Genesys, and has received honoraria from Cell Genesys for speaking engagements.

BACKGROUND. This open-label, multicenter, dose-escalation study evaluated multiple dose levels of immunotherapy in patients with metastatic hormone-refractory prostate cancer (HRPC). The immunotherapy, based on the GVAX platform, consisted of 2 allogeneic prostate-carcinoma cell lines modified to secrete granulocyte-macrophage-colony-stimulating factor (GM-CSF).

METHODS. Dose levels ranged from $100 \times 10^{6}$ cells q28d $\times 6$ to $500 \times 10^{6}$ cells prime $/ 300 \times 10^{6}$ cells boost q14d $\times 11$. Endpoints included safety, immunogenicity, overall survival, radiologic response, prostate-specific antigen (PSA) kinetics, and serum GM-CSF pharmacokinetics.

RESULTS. Eighty men, median age 69 years (range, 49-90 years), were treated. The most common adverse effect was injection-site erythema. Overall, the immunotherapy was well tolerated. A maximal tolerated dose was not established. The median survival time was 35.0 months in the high-dose group, 20.0 months in the mid-dose, group, and 23.1 months in the low-dose group. PSA stabilization occurred in 15 (19\%) patients, and a $>50 \%$ decline in PSA was seen in 1 patient. The proportion of patients who generated an antibody response to 1 or both cell lines increased with dose and included 10 of 23 (43\%) in the low-dose group, 13 of $18(72 \%)$ in the mid-dose group, and 16 of $18(89 \%)$ in the high-dose group ( $P$ $=.002$; Cochran-Armitage trend test).

CONCLUSIONS. This immunotherapy was well tolerated. Immunogenicity and overall survival varied by dose. Two phase 3 trials in patients with metastatic HRPC are underway. Cancer 2008;113:975-84. ㄷ 2008 American Cancer Society.

KEYWORDS: immunotherapy, granulocyte-macrophage-colony-stimulating factor, hormone-refractory prostate cancer, treatment, clinical trial, GVAX.

$\mathbf{0}$ ngoing research in cancer immunology has generated treatments that attempt to stimulate the patient's immune system to destroy cancer cells. ${ }^{1,2}$ Active immunotherapies in development for advanced prostate cancer have used different strategies to introduce single or multiple tumor-associated antigens in conjunction with cytokines or other immunostimulants intended to amplify the

The authors acknowledge the patients and families who participated in this clinical trial, the investigators and study coordinators, Tim Pinkerton and Jia Ma for statistical analyses, Laurie Wong for immunoblot analyses, and Michael Amantea for GM-CSF pharmacokinetic analyses.

All patients provided informed consent.
Address for reprints: Celestia S. Higano, MD, Seattle Cancer Care Alliance, 825 Eastlake Ave. E, P.0. Box 19023, Seattle, WA 981091023; Fax: (650) 595-0180; E-mail: todd@ mclarentodd.com

Received December 14, 2007; revision received April 9, 2008; accepted April 11, 2008. 
immune response. The GVAX platform of immunotherapies involves injection of whole tumor cells to provoke an immune response to multiple antigens expressed by the tumor cell, increasing the likelihood that the immune response will be relevant to the diversity of cancer cells existing in patients with advanced tumors. ${ }^{3}$ The tumor-cell lines are genetically modified to secrete granulocyte-macrophage-colony-stimulating factor (GM-CSF), an immune stimulant. Another approach involves autologous dendritic cells, loaded ex vivo with a fusion protein comprised of a single tumor-associated antigen, prostatic acid phosphatase, linked to GM-CSF. This single-antigen approach is patient-specific and requires collection of dendritic cells from individual patients by apheresis. ${ }^{4,5} \mathrm{~A}$ third approach involves injection of genetically modified viruses containing prostate-specific antigen (PSA). One or more genes that code for immunostimulants are loaded onto the virus to augment immunogenicity. ${ }^{6}$ The latter 2 strategies target single-tumor antigens that may be heterogeneously expressed among different patients and among different metastatic tumor sites in 1 patient. Overall, these immunotherapies have been well tolerated, and clinical trials are ongoing. ${ }^{2}$

Recent studies in a murine tumor model have provided insight into the mechanism of action of the GVAX platform of immunotherapies. ${ }^{7}$ GM-CSFsecreting tumor cells persisted and secreted high levels of GM-CSF at the injection site for more than 21 days, which resulted in dense infiltrates of dendritic cells. Such effects were not observed in mice treated with unmodified tumor cells administered alone or coadministered with injections of GM-CSF protein. An increase in activated dendritic cells and tumor antigen-specific T-cells was observed in draining lymph nodes of mice who received GM-CSF-modified tumor cells. These T-cell responses were directed against multiple tumor-associated antigens concurrently and induced protective immunity against multiple tumor-cell subclones of related, but antigenically distinct, tumors. These findings indicate that a whole tumor-cell immunotherapy approach has the potential to induce a broad-based immune response that is able to control antigenically distinct lesions common in metastatic tumors.

In an earlier trial that evaluated GVAX platform-based immunotherapy, GM-CSF-modified, allogeneic, prostate tumor cells (PC-3 and LNCaP) were administered to 34 patients with metastatic hormone-refractory prostate cancer (HRPC). This trial demonstrated a complete PSA response (PSA level dropped to $0.1 \mathrm{ng} / \mathrm{mL}$ ) in 1 patient, a reduced
PSA velocity in $73 \%$ of patients, stablized or decreased levels of a biomarker of osteolytic activity in $69 \%$ of patients, and produced median survival times of 34.9 and 24.0 months with the high and low doses of immunotherapy, respectively. ${ }^{8}$ The immunotherapy was subsequently modified to increase GM-CSF production. A phase 1-2, multicenter, openlabel study was designed to characterize the safety and activity of this modified product in patients with metastatic HRPC.

\section{MATERIALS AND METHODS}

This study was conducted according to the Helsinki Declaration, the Good Manufacturing Practices (GMP), and the National Institutes of Health (NIH) containment guidelines for recombinant DNA. The protocol was approved by each site's investigational review board. All patients signed an informed consent.

\section{Patients}

Men aged $\geq 18$ years with metastatic, histologically confirmed adenocarcinoma of the prostate were eligible. All patients were hormone refractory, defined as at least 2 successive increases in serum PSA $(\geq 2$ $\mathrm{ng} / \mathrm{mL}$ ) taken $\geq 2$ weeks apart, in the setting of castrate levels of testosterone. All patients were asymptomatic, chemotherapy-naive, and were receiving ongoing androgen deprivation therapy with a luteinizing hormone-releasing hormone (LHRH) analogue or had had an orchiectomy. Patients on combined androgen-blockade therapy underwent antiandrogen withdrawal before their study entry. Patients were excluded when they had transitional-cell, small-cell, neuroendocrine, or squamous-cell prostate cancer; had never responded to hormone therapy for prostate cancer; had cancer-related bone pain at screening; had uncontrolled medical conditions; had brain metastases; were on current treatment with bisphosphonates; had had prior gene therapy, chemotherapy, or immunotherapy; or use within 4 weeks of the study of systemic corticosteroid, biologic therapy, and/or radiation treatment.

\section{Study Design}

The immunotherapy was based on the GVAX platform (Cell Genesys, South San Francisco, Calif). Two cultured, allogeneic, prostate-cancer cell lines, LNCaP and PC-3, were genetically modified with a recombinant adeno-associated viral vector that encodes the human GM-CSF gene and irradiated to prevent cell division. Manufacturing was conducted 
TABLE 1

Dose Groups

\begin{tabular}{llll}
\hline Dose Group & Dose Level & Dose* & Schedule \\
\hline Low $(\mathrm{n}=33)$ & $1(\mathrm{n}=3)$ & $100 \times 10^{6}$ cells & $\mathrm{q} 28 \mathrm{~d} \times 6$ \\
& $2(\mathrm{n}=30)$ & $200 \times 10^{6}$ cells & $\mathrm{q} 28 \mathrm{~d} \times 6$ \\
Mid $(\mathrm{n}=25)$ & $3(\mathrm{n}=25)$ & $200 \times 10^{6}$ cells & q14d $\times 12$ \\
High $(\mathrm{n}=22)$ & $4(\mathrm{n}=3)$ & $300 \times 10^{6}$ cells & q14d $\times 12$ \\
& $5(\mathrm{n}=19)$ & $500 \times 10^{6}$ cells & $\times 1$ \\
& & $300 \times 10^{6}$ cells & q14d $\times 11$ \\
\hline
\end{tabular}

*Each dose was 50\% modified LNCaP and 50\% modified PC-3 cells.

according to cGMP and NIH containment guidelines for recombinant DNA.

The study included 5 dose levels (Table 1). Equal quantities of each cell line (half the total dose) were administered at each outpatient treatment visit to different intradermal injection sites. Dose levels were based on earlier trials of GVAX platform-based immunotherapies, which demonstrated that 100-500 million cell doses were well tolerated and immunologically active. ${ }^{8-10}$

Patients were sequentially assigned to escalating dose levels in groups of 3 to assess the safety of this immunotherapy. Further dose escalation was not feasible because of the number of injections required. After a safety evaluation, cohorts were expanded at Dose Level $2(\mathrm{n}=30)$ to evaluate a 28 -day treatment cycle, Dose Level $3(n=25)$ to evaluate a 14-day cycle, and Dose Level $5(n=19)$ to evaluate a high dose prime-boost schedule. Patients were treated for up to 6 months and followed for 1 year from the start of treatment or until they started a new treatment for prostate cancer. All patients were followed annually for life for potential late toxicities due to therapy. An amendment permitted 3 patients at Dose Level 5 to receive ongoing monthly maintenance injections.

\section{CLINICAL EVALUATION}

The study endpoints included safety, pharmacokinetics of serum GM-CSF, PSA response, ${ }^{11}$ radiologic tumor response, ${ }^{12}$ time to progression (PSA, radiologic), and overall survival. Patients who did not meet criteria for a complete and-or partial response or progressive disease for $\geq 90$ days were considered stable. Exploratory endpoints included analysis of PSA kinetics and systemic immune responses. PSA was tested at a central laboratory (Abbott AxSYM) at 2-week to 4-week intervals during treatment and follow-up. Computed tomography (CT) scans and chest $\mathrm{x}$-rays were performed at screening and at Months 3 , 6,9 , and 12. An optional substudy assayed GM-CSF levels on Days 1, 2, 3, 4, and 7 of several cycles. GMCSF concentration was measured by ELISA with the Quantikine HS GM-CSF Immunoassay kit (R\&D Systems, Minneapolis, Minn). Antibody responses were measured at baseline and at Weeks 12 and 24 by immunoblot analyses (2D electrophoresis) by using lysates of the modified immunotherapy cell lines blotted against patient sera. ${ }^{3,8}$ Exploratory analysis of a post-treatment $250 \mathrm{kD}$ band was conducted by immunoblot of the modified cell lines against a mouse monoclonal antifilamin-B probe and by direct immunoblot analysis of filamin-B protein against patient sera. Adverse events were coded by using the Medical Dictionary for Regulatory Activities (MedDRA, version 8.1), and graded by the National Cancer Institute's (NCI) Common Toxicity Criteria (CTC version 2).

\section{Statistical Analysis}

This study was designed to allow no more than 1 in 3 patients in the dose-escalation cohorts to experience dose-limiting toxicities (DLTs). Expansion cohorts were included to further evaluate safety and signals of activity. The 5 dose-level cohorts were pooled into 3 dose groups of comparable dose intensity to allow more meaningful interpretation of results. Summary statistics were gathered for the low-dose, mid-dose, and high-dose groups, and for all patients. Immunogenicity across dose groups was compared by using the Cochran-Armitage trend test. ${ }^{13}$ The Kaplan-Meier method was used to estimate the time to clinical endpoints. ${ }^{14}$ Patients who had not reached an endpoint by the date of analysis were censored on their last date of contact. WinNonLin version 3.1 (Pharsight, Mountain View, Calif) was used to estimate the GM-CSF pharmacokinetic parameters. The area under the curve (AUC) was calculated by using the linear trapezoidal method.

For exploratory analysis of PSA-slope data, a linear mixed-effects model was fitted to pretreatment and post-treatment PSA data, including all PSA data within 9 months pretreatment and 1 year post-treatment; PSA slopes pretreatment and post-treatment for each patient were estimated based on the model. Wilcoxon rank sum test was used to compare the PSA slopes between dose groups. Wilcoxon signed rank test was used to compare pretreatment and post-treatment slopes within each dose group.

Exploratory analyses evaluated the association of survival with clinical endpoints while controlling for dose group. The log-rank test was used to compare survival probability between groups. A Cox regression 
TABLE 2

Baseline Demographics

\begin{tabular}{|c|c|c|c|c|}
\hline & Low-Dose & Mid-Dose & High-Dose & All Patients \\
\hline & No. (\%) & No. (\%) & No. (\%) & No. (\%) \\
\hline No. of Patients & 33 & 25 & 22 & 80 \\
\hline \multicolumn{5}{|l|}{ Gleason score } \\
\hline $2-4$ & $1(3.0)$ & $1(4.0)$ & $1(4.5)$ & $3(3.8)$ \\
\hline $5-7$ & $16(48.5)$ & $6(24.0)$ & $10(45.5)$ & $32(40.0)$ \\
\hline $8-10$ & $15(45.5)$ & $15(60.0)$ & $8(36.4)$ & $38(47.5)$ \\
\hline Age, median [range] & $69[49-86]$ & $68[49-90]$ & $72[53-84]$ & $69[49-90]$ \\
\hline \multicolumn{5}{|l|}{ ECOG status } \\
\hline 0 & $23(69.7)$ & $18(72.0)$ & $18(81.8)$ & $59(73.8)$ \\
\hline 1 & $10(30.3)$ & $7(28.0)$ & $4(18.2)$ & $21(26.3)$ \\
\hline PSA, mean ng/mL [range] & $63[8-5873]$ & $37[1-2040]$ & $28[6-2107]$ & $51[1-5873]$ \\
\hline LDH, mean U/L [range] & $200[107-474]$ & 188 [126-325] & $174[130-229]$ & $189[107-474]$ \\
\hline Alkaline phosphatase, mean U/L [range] & $88[47-1283]$ & $78[54-326]$ & 78 [30-249] & 85 [30-1283] \\
\hline Hemoglobin, mean g/dL [range] & $13.3[9.7-15.2]$ & $12.5[8.4-14.9]$ & $13.2[11.6-15.1]$ & $13.1[8.4-15.2]$ \\
\hline \multicolumn{5}{|l|}{ Ethnicity } \\
\hline Caucasian & $31(93.9)$ & $24(96.0)$ & $18(81.8)$ & $73(91.3)$ \\
\hline African American & $0(0.0)$ & $1(4.0)$ & $1(4.5)$ & $2(2.5)$ \\
\hline Asian & $1(3.0)$ & $0(0.0)$ & $2(9.1)$ & $3(3.8)$ \\
\hline \multicolumn{5}{|l|}{ Site(s) of metastases } \\
\hline Lymph node & $12(36.4)$ & $11(44.0)$ & $11(50.0)$ & $34(42.5)$ \\
\hline Bone & $18(54.5)$ & $13(52.0)$ & $8(36.4)$ & $39(48.8)$ \\
\hline Lymph node+bone & $0(0.0)$ & $1(4.0)$ & $2(9.1)$ & $3(3.8)$ \\
\hline Other & $3(9.1)$ & 0 & $1(4.5)$ & $4(5.0)$ \\
\hline
\end{tabular}

ECOG indicates Eastern Cooperative Oncology Group; PSA, prostate-specific antigen.

model was used to obtain hazard ratios. The observed median survival time was compared with the median of predicted survival times calculated for each patient on the basis of baseline characteristics (including PSA, alkaline phosphatase, hemoglobin, lactate dehydrogenase, Gleason score, performance status, and visceral disease status) following a validated pretreatment prognostic model. ${ }^{15}$ Version 9.1 of SAS software (SAS Institute, Cary, NC) was used for data analyses.

\section{RESULTS \\ Patients}

Eighty patients with Stage IV (TX NX M1) metastatic HRPC were treated at 12 study sites. Although cohorts were not randomized, and the range in individual baseline values was large, mean and median values for baseline variables were not markedly different across the 3 dose groups (Table 2). When these baseline prognostic variables were used to calculate expected survival based on the Halabi pretreatment prognostic model, the predicted median survival times for the 3 dose groups were 18, 20, and 22 months, respectively.
Twenty-nine of 80 (36\%) patients received all scheduled treatments, including 14 of 33 (42\%) patients in the low-dose group, 7 of 25 (28\%) patients in the mid-dose group, and 9 of $22(41 \%)$ patients in the high-dose group. The median number of scheduled treatments received was 6 (range, 1-12 treatments), with a median of 4 (range, 1-6 treatments), monthly treatments in the low-dose group, 7 (range, 3-12 treatments) biweekly treatments in the middose group, and 7 (range, 2-12 treatments) biweekly treatments in the high-dose group. Three patients in the high-dose group also received a median of 9 (range, 6-21 treatments) monthly maintenance treatments. The median duration of treatment was 86 days in the low-dose group, 81 days in the mid-dose group, and 85 days in the high-dose group. The primary reason for stopping the study (6 months of treatment plus 6 months follow-up) prematurely was progressive disease in $65(90 \%)$ patients, an adverse event in $2(2.8 \%)$ patients, consent withdrawal in 2 (2.8\%) patients, death in $2(2.8 \%)$ patients, and other in $1(1.4 \%)$ patient. Of those withdrawn for disease progression, $75 \%$ had radiologic progression, $19 \%$ had PSA progression, and $6 \%$ had both. After stopping the study, $66(82.5 \%)$ patients received additional therapy for prostate cancer within 1 year of 


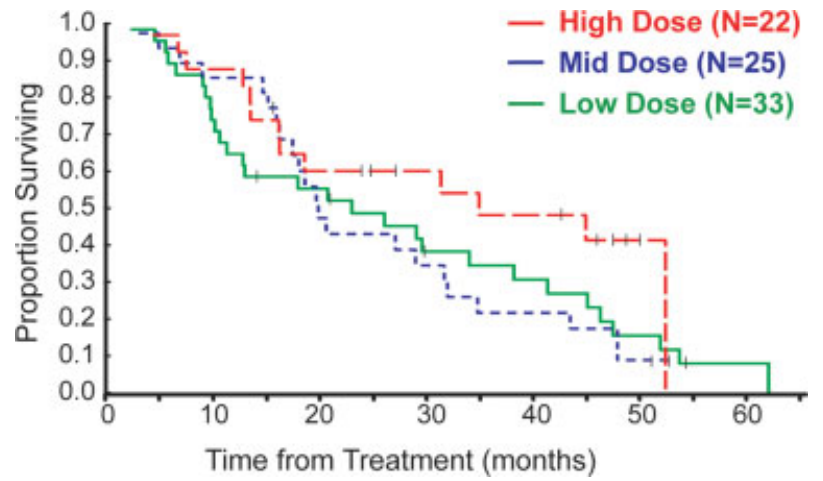

FIGURE 1. The Kaplan-Meier estimate of overall survival time is illustrated. Patients who had not died at the time of data analysis are indicated by hash marks.

starting therapy including 29 of $33(88 \%)$ in the lowdose group, 20 of $25(80 \%)$ in the mid-dose group, and 17 of $22(87 \%)$ in the high-dose group. Such therapy included radiation in 10 of 80 (12.5\%), hormonal therapy in 9 of 80 (11.3\%), nondocetaxel chemotherapy in 9 of 80 (11\%), and docetaxel-based chemotherapy in 15 of 80 (19\%). As of 50 months after the last patient was enrolled, $9(11.3 \%)$ patients were alive, $64(80.0 \%)$ had died, and $7(8.8 \%)$ were lost to follow-up.

\section{Survival}

Median survival time after treatment initiation was 23.1 months in the low-dose group, 20.0 months in the mid-dose group, and 35.0 months in the highdose group (Fig. 1). The observed median survival time (Kaplan-Meier method) exceeded the predicted survival time (Halabi pretreatment prognostic model) in the low-dose group (23.1 months vs 18.0 months) and the high-dose group (35.0 months vs 22.0 months) and met the predicted survival time in the mid-dose group (20.0 months vs 20.0 months). ${ }^{14,15}$

\section{IMMUNOGENICITY}

Immunoblot analyses of lysates of the 2 modified cell lines, PC-3 and LNCaP, against patient sera were performed at baseline and at post-treatment to assess induction of antibody responses against the prostate cancer cells. Immunoblot data were available at baseline and at least 1 post-treatment time point in 61 of $80(76.2 \%)$ patients, including 59 at Week 12, 36 at Week 24, and 34 at all 3 time points. The proportion of patients who developed new or enhanced immunoreactive bands compared with baseline increased with dose at Week $12(P=.002$; Cochran-Armitage trend test) and over time (Fig. 2).

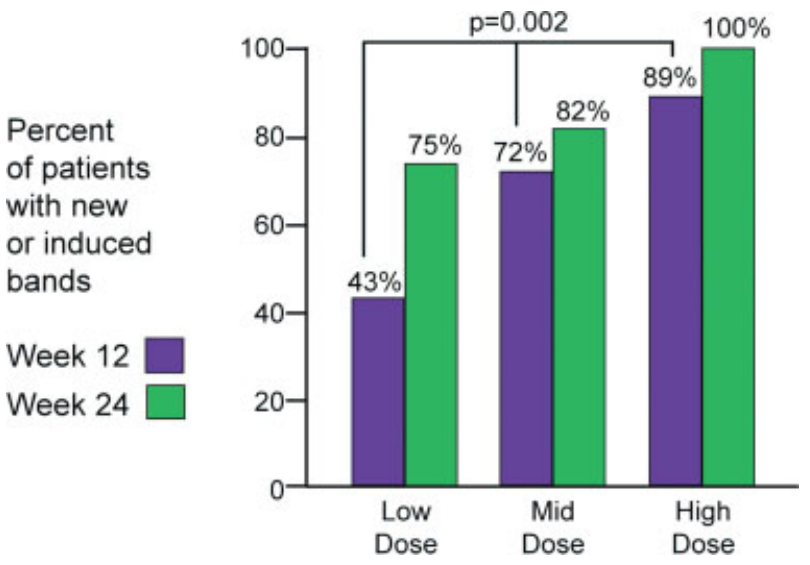

FIGURE 2. This is the proportion of patients who were immunoreactive to at least 1 cell line at Week 12 and Week 24.

Immunoreactivity was variable with patients mounting antibodies to either 1 or both cell lines (Table 3). Of the 20 patients without evidence of antibody response to at least 1 modified cell line at Week 12 , 10 patients subsequently developed new bands at Week 24 (Table 3).

Development of antibodies to either cell line at Week 12 did not appear to be associated with longer survival. However, the induction of antibodies to 1 or both modified cell lines at Week 24 was associated with a $29 \%$ decrease in hazard rate; the median survival for these 30 patients was 34 months compared with 16 months for those 6 patients without induced antibody responses (HR, 0.71; 95\% confidence interval $[\mathrm{CI}], 0.22-2.31 ; P=.57$; log-rank test; adjusted for dose group).

The most frequently occurring new or enhanced immunoreactive band $(\approx 250 \mathrm{kD})$ compared with baseline occurred in immunoblots of 23 of 61 (38\%) patients at Week 12 or Week 24, including 8 of 25 (32\%) immunoblots in the low-dose group, 7 of 18 (39\%) in the mid-dose group, and 8 of 18 (44\%) in the high-dose group. The median survival time was 35 months in the 23 patients with the $250 \mathrm{kD}$ band at either Week 12 or Week 24 compared with 29 months in the 38 patients who did not exhibit a new or enhanced $250 \mathrm{kD}$ band at any time point (HR, 0.72; 95\% CI; 0.39-1.33; $P=.29$; log-rank test; adjusted for dose group). For the 34 patients with data at both Week 12 and Week 24, the median survival time was 48 months in the 8 patients with a $250 \mathrm{kD}$ band present at both time points compared with 29 months in the other 26 patients with the 250 $\mathrm{kD}$ band at only 1 or no time point (HR, 0.46 ; $95 \%$ CI; 0.16-1.30; $P=.13$; log-rank test; adjusted for dose group). Immunoblot analyses of modified immu- 
TABLE 3

Immunogenicity

\begin{tabular}{|c|c|c|c|c|}
\hline & Low-Dose & Mid-Dose & High-Dose & All Patients \\
\hline & №. (\%) & №. (\%) & No. (\%) & No. (\%) \\
\hline No. of Patients & 33 & 25 & 22 & 80 \\
\hline \multicolumn{5}{|l|}{ Week 12} \\
\hline No. with immunoblot data & 23 & 18 & 18 & 59 \\
\hline No. who developed antibodies against PC-3 and/or LNCaP & $10 / 23(43)$ & $13 / 18(72)$ & $16 / 18(89)$ & $39 / 59(66)$ \\
\hline No. who developed antibodies against PC-3 & 9/23 (39) & $11 / 18(61)$ & $16 / 18(89)$ & $36 / 59(61)$ \\
\hline No. who developed antibodies against LNCaP & $6 / 23(26)$ & $10 / 18(56)$ & $12 / 18(67)$ & $28 / 59(48)$ \\
\hline No. who developed antibodies against both & $5 / 23(22)$ & 8/18 (44) & $12 / 18(67)$ & $25 / 59(42)$ \\
\hline \multicolumn{5}{|l|}{ Week 24} \\
\hline No. with immunoblot data & 16 & 11 & 9 & 36 \\
\hline No. who developed antibodies against PC-3 and/or LNCaP & $12 / 16(75)$ & 9/11 (82) & $9 / 9(100)$ & $30 / 36(83)$ \\
\hline No. who developed antibodies against PC-3 & $10 / 16(62)$ & $7 / 11(64)$ & $9 / 9(100)$ & $26 / 36(72)$ \\
\hline No. who developed antibodies against $\mathrm{LNCaP}$ & $8 / 16(50)$ & $7 / 11(64)$ & $8 / 9(89)$ & $23 / 36(64)$ \\
\hline No. who developed antibodies against both & $6 / 16(38)$ & $5 / 11(46) \%$ & $8 / 9(89)$ & $19 / 36(53)$ \\
\hline \multicolumn{5}{|l|}{ Weeks 12 and 24} \\
\hline No. with immunoblot data & 14 & 11 & 9 & 34 \\
\hline No. who developed antibodies against PC-3 and/or LNCaP at Week 12 & $7 / 14(50)$ & $7 / 11(64)$ & $9 / 9(100)$ & $23 / 34(68)$ \\
\hline No. who developed antibodies against both cell lines at Week 12 & $4 / 14(29)$ & $3 / 11(27)$ & $7 / 9(78)$ & $14 / 34(41)$ \\
\hline No. with no response to at least 1 cell line at Week 12 & 10 & 8 & 2 & 20 \\
\hline No. who developed antibodies at Week 24 against a cell line to & & & & \\
\hline which they had no response at Week 12 & $5 / 10(50)$ & $4 / 8(50)$ & $1 / 2(50)$ & $10 / 20(50)$ \\
\hline
\end{tabular}

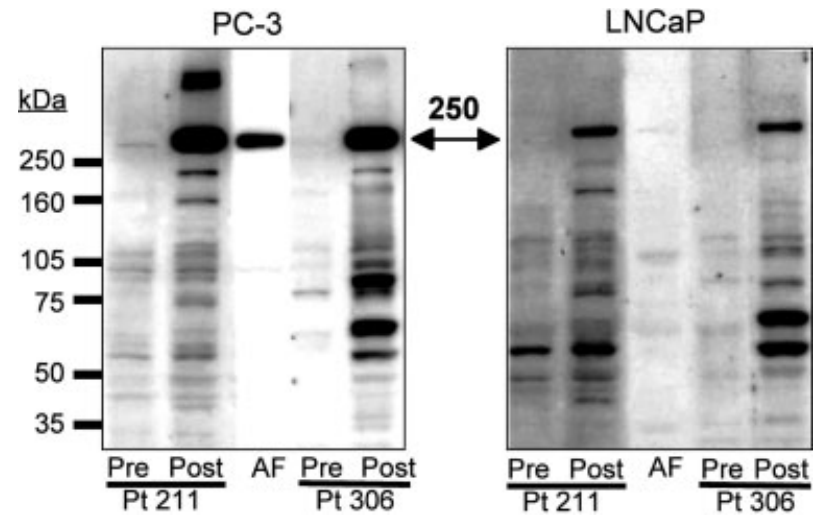

FIGURE 3. Depicted is an immunoblot analysis of modified PC-3 and LNCaP cell lysates that show comigration of immunoreactive bands (250 kD) with sera from 2 patients and with a mouse monoclonal antifilamin (AF) probe.

notherapy cell lysates showed comigration of immunoreactive bands $(250 \mathrm{kD})$ with sera from 2 patients and a mouse monoclonal antifilamin (AF) probe. The band was identified as filamin-B by direct immunoblot analysis of filamin protein against patient sera (Fig. 3).

\section{Serum GM-CSF Levels}

The optional GM-CSF substudy included 23 patients, 6 in the low-dose group and 17 in the high-dose group. Serum GM-CSF data were obtained during 18 treatment cycles in the low-dose group and during 32 cycles in the high-dose group. Peak GM-CSF values were generally observed 1 day (range, 1-3 days) post-treatment, with peak serum levels of 1.7$15.3 \mathrm{pg} / \mathrm{mL}$ in the low-dose group and 4.7-579.9 pg/ $\mathrm{mL}$ in the high-dose group. Peak values declined with subsequent dosing in all but 4 patients.

Serum GM-CSF AUC values, a measure of total exposure, exhibited a similar pattern to GM-CSF peak serum levels, with higher AUCs seen after the first dose compared with subsequent doses, and the highest AUCs occurred in the high-dose group (range, $2.34 \mathrm{pg} / \mathrm{mL}$ to $1614 \mathrm{pg} / \mathrm{mL} \cdot$ per day). Transient increases in white blood cells (range, 3.0 thous/ cu mm to 24.2 thous/cu mm), neutrophils (range, 1.5 thous/cu $\mathrm{mm}$ to 18.0 thous/cu $\mathrm{mm}$ ), and eosinophils (range, 0 thous/cu $\mathrm{mm}$ to 3.6 thous/cu $\mathrm{mm}$ ) were observed typically 1-2 days post-treatment, and all levels returned to baseline typically by Day 7 . Lymphocyte levels (range, 0.5 thous/cu mm to 5.8 thous/ cu $\mathrm{mm}$ ) remained unchanged.

In an analysis extrapolated from a murine model, GM-CSF AUCs in 7 patients surpassed 206 $\mathrm{pg} / \mathrm{mL}$.per day, a threshold level shown to induce myeloid suppressor cells in mice. ${ }^{16}$ These 7 values were observed only in the high-dose group during 32 treatment cycles. Six occurred in the first treatment 


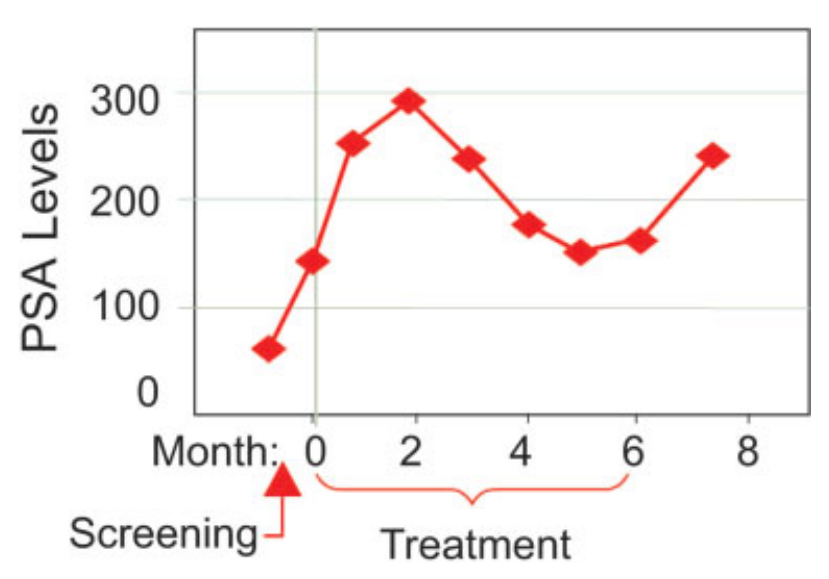

FIGURE 4. Serum PSA over time is shown for a patient with a delayed decline in PSA post-treatment.

cycle and 1 in the third, with all other values obtained for each individual patient falling below the threshold. No GM-CSF AUC measurements above this threshold were observed among the 18 recorded treatment cycles in the low-dose group.

\section{Serum PSA Changes}

One $(3.0 \%)$ patient in the low-dose group $(n=33)$ had a PSA reduction of $>50 \%$ lasting 3.9 months. Stable disease (PSA decline of $<50 \%$ or increase of $<25 \%)$ lasting for at least 90 days occurred in 15 patients, including 4 of 33 (12.1\%) in the low-dose, 6 of $25(24.0 \%)$ in the mid-dose and 5 of $22(22.7 \%)$ in the high-dose group. The median time to PSA progression was 2.8 months in the low-dose, 2.2 months in the mid-dose group, and 2.5 months in the highdose group. There were no significant differences between pretreatment and post-treatment PSA slopes within or between dose groups. A negative deflection of any magnitude in PSA slope post-treatment was observed for 42 of 80 (52.5\%) patients. These 42 patients had a median survival of 31 months versus 19 months in those without negative deflection (HR, $0.65 ; 95 \% \mathrm{CI}, 0.38-1.10 ; P=.11$, log-rank test; adjusted for dose group). Many patients had variable PSA patterns with multiple slope changes, including some with a decline after an initial increase (Fig. 4).

\section{Radiologic Response}

Of the 80 treated patients, $28(35.0 \%)$ had an investigator-assessed radiologic response of stable disease, including $16(49 \%)$ in the low-dose, $6(24 \%)$ in the mid-dose and $6(27 \%)$ in the high-dose group. None had an objective radiologic response. Patients with stable disease had a median survival time of 43 months compared with 19 months in those who pro- gressed (HR, 0.43; 95\% CI, 0.24-0.77; $P=.004$, logrank test; adjusted for dose group). The median time to clinical progression or death was 2.8 months $(95 \%$ CI, 2.8-3.3 months) and was similar across dose groups. New bone pain was reported as an adverse event by 23 (28.8\%) patients, including 10 in the lowdose group, 9 in the mid-dose group, and 4 in the high-dose group. The median onset of bone pain was 2.1 months (range, 0.3-7.8 months) overall, 2.6 months in the low-dose group, 2.2 months in the mid-dose group, and 0.4 months in the high-dose group.

\section{Safety}

A maximal tolerated dose was not identified. The frequency of unsolicited, treatment-related, adverse events was similar across dose groups and included nausea in 5 (6.3\%) patients, anorexia in $3(3.8 \%)$, anemia, thrombocytopenia, dyspnea, fatigue, peripheral edema, and weight loss in 2 (2.5\%) patients. Injection-site reactions occurred in all patients (100\%), including erythema, induration, pruritus, and pain or soreness. The majority of adverse events were grade 1 or grade 2. Four patients had grade 3 or grade 4 adverse events that were considered related to treatment, including fatigue, deep vein thrombosis, thrombocytopenia, anemia, subdural hematoma, and weight loss. Four patients died during the treatment phase, attributed to progressive disease $(\mathrm{n}=$ $2)$, chronic obstructive lung disease $(n=1)$, and unknown cause $(n=1)$. No evidence of autoimmune disease was observed.

Adverse events commonly associated with immunotherapy were actively solicited from patients and were consistent with flu-like symptoms (Table 4). Investigators were not asked to assign causality for these events.

\section{DISCUSSION}

This multicenter, open-label trial was undertaken to evaluate the safety, immunogenicity, and clinical activity of escalating doses of a GM-CSF-secreting, allogeneic, cellular immunotherapy. All patients had metastatic hormone-refractory prostate cancer (HRPC) and were chemotherapy-naive. Patients had a range of baseline characteristics that fell within the ranges of typical metastatic HRPC patients as seen in the Halabi Learning Sample and the TAX 327 Study. ${ }^{15,17}$ The immunotherapy was well tolerated at all dose levels, and no maximal tolerated dose (MTD) was established in this trial. The most common adverse effect was injection-site reaction. Numerous findings in this trial support use of a high 
TABLE 4

Solicited Systemic Reactions

\begin{tabular}{|c|c|c|c|c|c|}
\hline & & Low-Dose $n=33$ & Mid-Dose $n=25$ & High-Dose $n=22$ & All Patients $n=80$ \\
\hline & & №. (\%) & No. (\%) & No. $(\%)$ & №. (\%) \\
\hline \multirow[t]{2}{*}{ Fatigue } & Mild-moderate & $26(78.8)$ & $14(56.0)$ & $17(77.3)$ & $57(71.3)$ \\
\hline & Severe & $4(12.1)$ & $8(32.0)$ & $3(13.6)$ & $15(18.8)$ \\
\hline \multirow[t]{2}{*}{ Headache } & Mild-moderate & $18(54.5)$ & $14(56.0)$ & $10(45.5)$ & $42(52.5)$ \\
\hline & Severe & 0 & $1(4.0)$ & $1(4.5)$ & $2(2.5)$ \\
\hline \multirow[t]{2}{*}{ Malaise } & Mild-moderate & $17(51.5)$ & $10(40.0)$ & $12(54.5)$ & $39(48.8)$ \\
\hline & Severe & $3(9.1)$ & $6(24.0)$ & $4(18.2)$ & $13(16.3)$ \\
\hline \multirow[t]{2}{*}{ Myalgia } & Mild-moderate & $11(33.3)$ & $14(56.0)$ & $13(59.1)$ & $38(47.5)$ \\
\hline & Severe & $4(12.1)$ & $3(12.0)$ & $2(9.1)$ & $9(11.3)$ \\
\hline \multirow[t]{2}{*}{ Arthralgia } & Mild-moderate & $16(48.5)$ & $10(40.0)$ & $12(54.5)$ & $38(47.5)$ \\
\hline & Severe & $1(3.0)$ & $3(12.0)$ & $2(9.1)$ & $6(7.5)$ \\
\hline \multirow[t]{2}{*}{ Systemic pruritus } & Mild-moderate & $12(36.4)$ & $9(36.0)$ & $14(63.6)$ & $35(43.8)$ \\
\hline & Severe & $1(3.0)$ & $1(4.0)$ & 0 & $2(2.5)$ \\
\hline \multirow[t]{2}{*}{ Chills } & Mild-moderate & $10(30.3)$ & $9(36.0)$ & $12(54.5)$ & $31(38.8)$ \\
\hline & Severe & $1(3.0)$ & $1(4.0)$ & 0 & $2(2.5)$ \\
\hline \multirow[t]{2}{*}{ Systemic rash } & Mild-moderate & $6(18.2)$ & $5(20.0)$ & $10(45.5)$ & $21(26.3)$ \\
\hline & Severe & $1(3.0)$ & 0 & 0 & $1(1.3)$ \\
\hline \multirow[t]{2}{*}{ Fever } & Mild-moderate & $5(15.2)$ & $6(24.0)$ & $3(13.6)$ & $14(17.5)$ \\
\hline & Severe & 0 & 0 & 0 & 0 \\
\hline \multirow[t]{2}{*}{ Hives } & Mild-moderate & $5(15.2)$ & $3(12.0)$ & $5(22.7)$ & $13(16.3)$ \\
\hline & Severe & 0 & 0 & 0 & 0 \\
\hline \multirow[t]{2}{*}{ Rigors } & Mild-moderate & $3(9.1)$ & $4(16.0)$ & $5(22.7)$ & $12(15.0)$ \\
\hline & Severe & $1(3.0)$ & $1(4.0)$ & 0 & $2(2.5)$ \\
\hline
\end{tabular}

dose of immunotherapy in phase 3 trials, including a favorable safety profile, immunogenicity, GM-CSF levels, and survival times.

Serum GM-CSF levels peaked 1-3 days after treatment. Transient increases in white blood cells, neutrophils, and eosinophils were observed, thereby confirming bioactivity of GM-CSF, and all levels returned to baseline before subsequent treatment. Lymphocyte levels remained unchanged. Peak serum GM-CSF levels did not exceed, and in most patients were 30-100-fold lower than, peak GM-CSF serum levels of $1.5-5 \mathrm{ng} / \mathrm{mL}$ reported in patients treated with the approved $250 \mu \mathrm{g} / \mathrm{m}^{2}$ dose of recombinant GM-CSF administered intravenously or subcutaneously. ${ }^{18}$ Similarly, the GM-CSF AUC was typically markedly lower than the AUC of approximately 365 to $460 \mathrm{pg} / \mathrm{mL}$-day measured in patients receiving subcutaneous or intravenous recombinant GM-CSF, respectively.

Although direct extrapolation from murine data may be limited in value because of species-specific differences in cytokine activity, the AUCs of serum GM-CSF in 7 patients had values above $206 \mathrm{pg} /$ $\mathrm{mL}$-day, a threshold at which GM-CSF-secreting immunotherapy has been shown to induce myeloid suppressor cells in mice. ${ }^{16}$ However, all subsequent and/or previous AUC values for each patient were below this threshold, suggesting that sustained induction of myeloid suppressor cells is unlikely.

Antibody responses to the modified LNCaP and PC-3 cell lines were associated both with cell dose and treatment duration. By 24 weeks, $75 \%, 82 \%$, and $100 \%$ of patients in the low-dose group, mid-dose group, and high-dose group, respectively, had developed reactive antibodies to the modified cell lines. The increase in the frequency of immune responses between Weeks 12 and 24 supports a minimum of 24 weeks of therapy; this represents the duration of initial treatment chosen for phase 3 trials.

In this study, 23 patients had an induced $250 \mathrm{kD}$ band on immunoblot, and the proportion of patients with this band increased with dose group. This band was found to comigrate with filamin-B, and subsequent evaluation in 2 patients identified the $250 \mathrm{kD}$ band as filamin-B. Filamin-B, a cytoskeletal protein prevalent in endothelial cells, plays a central role in cell shape, division, adhesion, motility, signal transduction, and protein sorting. ${ }^{19,20}$ Filamin-B has been linked to cancer through functional studies and has been identified as a candidate cancer gene in a study of mutation rates in breast and colorectal cancers. ${ }^{21}$ The presence of this $250 \mathrm{kD}$ band was associated 
with survival. This effect was most pronounced in patients who developed the antibody by Week 12 and sustained it at Week 24. In a prior study of this immunotherapy, induction of an antifilamin response was found in an HRPC patient whose PSA dropped to $0.1 \mathrm{ng} / \mathrm{mL}$ and who had resolution of a bone lesion. ${ }^{8}$

Similar to other trials of prostate cancer immunotherapy, objective PSA or radiologic responses are uncommon and were infrequent in this trial. However, a flattening (any negative deflection) in the slope of PSA was associated with numerically longer survival times as was investigator-assessed stable disease. Although PSA-slope changes and disease stabilization are not established as reliable outcome measures and may simply reflect more indolent disease, these endpoints may also capture the presumed mechanism of action of immunotherapy, with less immediate cytotoxicity but greater durability of effect. The use of objective PSA and radiologic responses as endpoints may be of less utility in immunotherapy trials than in chemotherapy trials, and survival, rather than PSA and radiologic response, will be the primary endpoint in future phase 3 trials of this immunotherapy.

Kaplan-Meier estimates of survival varied by dose group, with median survival times of 23, 20, and 35 months for the low-dose group, mid-dose group, and high-dose group, respectively. The result in the high-dose group is consistent with the observed median survival time of 34.9 months in the prior phase $1 / 2$ trial of this immunotherapy dose in a similar population of patients. ${ }^{8}$ On the basis of the Halabi pretreatment prognostic model, a predicted median survival time in each dose group was calculated by using baseline prognostic factors. ${ }^{15}$ Whereas differences in predicted median survival between dose groups likely reflect some heterogeneity in baseline risk factors, the actual median survival time of patients treated with immunotherapy numerically exceeded the expected survival times by 5 and 13 months in 2 of the 3 dose groups and matched the predicted survival in the third. These data suggest that the observed survival is not simply due to selection of good-risk patients for the trial. Although useful in terms of providing a context to interpret survival observations, this prognostic model may not accurately predict survival times when there are changes to the standard of care. Twenty percent of patients in this immunotherapy trial received subsequent docetaxel treatment during the year after the start of immunotherapy, and it is unknown how many may have received docetaxel treatment after the end of the active follow-up period.
Limitations of this study include the lack of a control arm; at the time this study was conducted, there was no established standard of care for metastatic HRPC. In addition, patients were enrolled sequentially rather than randomly, which was necessary because of the safety evaluation but may have resulted in an uneven distribution of patients. Immunogenicity analysis focused on the induction of humoral responses by established methodologies. In contrast, no established methodologies existed for monitoring of T-cell responses to an allogeneic whole tumor cell-based product for which the immunodominant antigens have not yet been identified. Finally, patients were followed for only 1 year from the start of immunotherapy; therefore, data on poststudy use of docetaxel is incomplete. Because docetaxel has been shown to improve survival duration in patients with HRPC, possible use of docetaxel in this patient population after the follow-up period might have affected the survival outcome.

In conclusion, the observations of dose-associated immunogenicity and encouraging overall survival rates in the absence of dose-limiting toxicity, as well as the consistency of these results with data from a previous trial, have led to initiation of 2 phase 3 trials that use the high dose of this GM-CSF-secreting, allogeneic, cellular immunotherapy. These phase 3 trials will confirm whether there is a survival benefit with this immunotherapy in men with metastatic hormone-refractory prostate cancer.

\section{REFERENCES}

1. Sanda MG, Ayyagari SR, Jaffee EM. Demonstration of a rational strategy for human prostate cancer gene therapy. $J$ Urol. 1994;151:622-628.

2. Schlom J, Arlen PM, Gulley JL. Cancer vaccines: moving beyond current paradigms. Clin Cancer Res. 2007;13:37763782.

3. Simons JW, Carducci MA, Mikhak B, et al. Phase I/II trial of an allogeneic cellular immunotherapy in hormone-naive prostate cancer. Clin Cancer Res. 2006;12(11 pt 1):33943401.

4. Small EJ, Schellhammer PF, Higano CS, et al. Placebocontrolled phase III trial of immunologic therapy with sipuleucel-T (APC8015) in patients with metastatic, asymptomatic hormone refractory prostate cancer. J Clin Oncol. 2006;24:3089-3094.

5. Lin AM, Hershberg RM, Small EJ. Immunotherapy for prostate cancer using prostatic acid phosphatase loaded antigen presenting cells. Urol Oncol. 2006;24:434-441.

6. Dipaola R, Plante M, Kaufman $\mathrm{H}$, et al. A phase I trial of pox PSA vaccines (PROSTVAC-VF) with B7-1, ICAM-1, and LFA-3 co-stimulatory molecules (TRICOM) in patients with prostate cancer. J Transl Med. 2006;4:1.

7. Simmons AD, Li B, Gonzalez-Edick M, et al. GM-CSFsecreting cancer immunotherapies: preclinical analysis of the mechanism of action. Cancer Immunol Immunother. 2007;56:1653-1665. 
8. Small EJ, Sacks N, Nemunaitis J, et al. GM-CSF-secreting, allogeneic cellular immunotherapy for hormone-refractory prostate cancer. Clin Cancer Res. 2007;13:3883-3891.

9. Jaffee EM, Hruban RH, Biedrzycki B, et al. Novel allogeneic granulocyte-macrophage colony-stimulating factor-secreting tumor vaccine for pancreatic cancer: a phase I trial of safety and immune activation. J Clin Oncol. 2001;19:145-156.

10. Thomas AM, Santarsiero LM, Lutz ER, et al. Mesothelinspecific $\mathrm{CD} 8(+) \mathrm{T}$ cell responses provide evidence of in vivo cross-priming by antigen-presenting cells in vaccinated pancreatic cancer patients. J Exp Med. 2004;200:297306.

11. Bubley GJ, Carducci M, Dahut W, et al. Eligibility and response guidelines for phase II clinical trials in androgenindependent prostate cancer: recommendations from the Prostate-Specific Antigen Working Group. J Clin Oncol. 1999;17:3461-3467. Erratum in: J Clin Oncol. 2000;18:2644.

12. Green S, Weiss GR. Southwest Oncology Group standard response criteria, endpoint definitions and toxicity criteria. Invest New Drugs. 1992;10:239-253.

13. Agresti A. Categorical Data Analysis, New York: John Wiley; 1990:100-102.

14. Kaplan EI, Meier P. Nonparametric estimation from incomplete observations. J Am Stat Assoc. 1958;53:457.
15. Halabi S, Small EJ, Kantoff PW, et al. Prognostic model for predicting survival in men with HRPC. J Clin Oncol. 2003;21:1232-1237.

16. Serafini P, Carbley R, Noonan KA, Tan G, Bronte V, Borrello I. High-dose granulocyte-macrophage colony-stimulating factor-producing vaccines impair the immune response through the recruitment of myeloid suppressor cells. Cancer Res. 2004;64:6337-6343.

17. Tannock IF, de Wit R, Berry WR, et al. Docetaxel plus prednisone or mitoxantrone plus prednisone for advanced prostate cancer. $N$ Engl J Med. 2004;15:1502-1512.

18. [Anonymous Author]. Leukine Sargramostim Package Insert, Revision 0230-03. Richmond, California: Berlex Laboratories; June 2002.

19. van der Flier A, Sonnenberg A. Structural and functional aspects of filamins. Biochim Biophys Acta. 2001;1538:99117.

20. Takafuta T, Wu G, Murphy GF, Shapiro SS. Human betafilamin is a new protein that interacts with the cytoplasmic tail of glycoprotein Ibalpha. J Biol Chem. 1998;273:1753117538.

21. Sjoblom T, Jones S, Wood LD, et al. The consensus coding sequences of human breast and colorectal cancers. Science. 2006;314:268-274. 